\title{
Fibromialgia: abordagem terapêutica
}

Paula Gomes, ${ }^{*}$ Carla Campos**

\section{RESUMO}

Objectivos: Rever, com base na evidência, e sistematizar a informação recentemente publicada sobre a abordagem terapêutica do doente com fibromialgia (FM).

Fontes de Dados: National Guideline Clearinghouse, Cochrane, Dare, Bandolier e Medline.

Métodos de Revisão: Pesquisa de normas de orientação clínica (NOC), meta-análises (MA), revisões sistemáticas (RS) e estudos originais, em língua inglesa e portuguesa, publicados entre 1998 e Setembro de 2008, utilizando as palavras-chave: «Fibromyalgia» e «Therapeutics». Foram também pesquisadas recomendações de sociedades científicas, tendo sido seleccionadas aquelas em que é analisado o nível de evidência. Foram incluídos 2 NOC, 3 MA, 11 RS e 4 estudos originais. Utilizou-se a taxonomia SORT para avaliação do nível de evidência e força de recomendação.

Resultados: A abordagem da FM requer uma avaliação da dor, funcionalidade, contexto psicossocial e co-morbilidades associadas. Relativamente à terapêutica farmacológica, existe forte evidência para a utilização de duloxetina, pregabalina e tramadol para o controlo da dor. Evidência moderada apoia a utilização de amitriptilina e ciclobenzaprina (especialmente se perturbações do sono associadas) e fluoxetina. O milnaciprano e o pirlindole apresentam resultados promissores, sendo necessários mais estudos. Os efeitos benéficos observados com a fluoxetina, duloxetina e milnaciprano foram independentes das alterações do humor e as doses utilizadas foram similares ou superiores às recomendadas para o tratamento anti-depressivo, conferindo-lhes a vantagem de tratarem depressões co-mórbidas. Em relação à terapêutica não farmacológica, existe evidência moderada para a prática de exercício físico aeróbico e balneoterapia.

Conclusões: Múltiplos tratamentos farmacológicos e não-farmacológicos demonstraram eficácia no tratamento da dor e sintomas associados. Na ausência de um tratamento que actue em todos os sintomas, um tratamento optimizado irá requerer uma abordagem multidisciplinar individualizada.

Palavras-chave: Fibromialgia; Tratamento; Dor.

\section{INTRODUÇÃO}

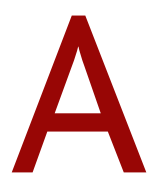
fibromialgia (FM) é uma síndrome de dor músculo-esquelética generalizada, crónica e de etiologia desconhecida, com elevado impacto na qualidade de vida e funcionalidade dos doentes, acarretando custos pessoais, sociais e de saúde significativos. ${ }^{1}$

A sua prevalência na população geral é de cerca de $2 \%$, estimando-se que represente 5 a $10 \%$ das Consultas de Medicina Geral e Familiar e 15\% das Consultas de Reumatologia. Afecta mais frequentemente o sexo feminino (relação homem-mulher de 1:6 a 1:10), com idade de início oscilando mais frequentemente entre os 20 e os 50 anos. $^{1}$

*Assistente de Medicina Geral e Familiar, USF Infesta

**Médica Interna de Medicina Geral e Familiar, Centro de Saúde de Leça da Palmeira
As dores difusas parecem relacionar-se com os tecidos moles - músculos, tendões, ligamentos - e não representam patologia intra-articular ou óssea. ${ }^{2}$ É acompanhada por sintomatologia sistémica incluindo, entre outras, alterações quantitativas e qualitativas do sono, fadiga, cefaleias, alterações cognitivas (como perda de memória e dificuldade de concentração), parestesias / disestesias, ansiedade e, em cerca de um terço dos casos, depressão. ${ }^{3}$

O seu diagnóstico é essencialmente clínico, baseado numa história de dor generalizada (definida como dor no lado esquerdo e direito do corpo, dor acima e abaixo da cintura e dor no esqueleto axial) durante pelo menos três meses, e pela presença de pontos dolorosos à pressão digital em áreas simétricas do corpo e com localização bem estabelecida (Quadro I). Embora seja necessária a presença de pelo menos 11 pontos dolorosos 
QUADRO I. Localização dos 18 pontos dolorosos a avaliar segundo com os critérios para a classificação da fibromialgia do Colégio Americano de Reumatologia (1990).

\section{LOCALIZAÇÃO DOS PONTOS DOLOROSOS}

- Cervical inferior: bilateral, na face anterior dos espaços intertransversários de $\mathrm{C} 5$ a $\mathrm{C7}$;

- Segunda costela: bilateral, imediatamente para fora da junção costocondral da $2^{a}$ costela e na face superior;

- Epicôndilo: bilateral, 2 cm externamente ao epicôndilo

- Joelho: bilateral, na almofada adiposa interna, acima da interlinha articular;

- Occipital: bilateral, nas inserções do músculo suboccipital;

- Trapézio: bilateral, no ponto médio do bordo superior do músculo;

- Supra-espinhoso: bilateral, na origem do músculo acima da espinha da omoplata junto do bordo interno;

- Glúteo: bilateral, no quadrante superior-externo da nádega no folheto anterior do músculo;

- Grande trocanter: bilateral, posterior à proeminência trocantérica.

em 18 possíveis para classificar esta síndrome de acordo com os critérios do Colégio Americano de Reumatologia (1990), aquele número pode não ser necessário para estabelecer o diagnóstico. São ainda de valorizar várias patologias que se podem sobrepor ou mimetizar a FM e que podem ser excluídas através da história clínica, do exame objectivo e de determinados meios complementares de diagnóstico.,3

A natureza e etiologia da FM suscitam ainda várias dúvidas e controvérsias. Sabe-se que os doentes com FM têm limiares de dor mais baixos e alterações centrais no processamento da dor, que foram evidenciadas em técnicas de imagem cerebral e pela presença de concentrações três vezes mais elevadas de substância P no líquido cerebroespinhal. Vários factores genéticos e neuroendócrinos são predisponentes para o desenvolvimento de FM. Os familiares em primeiro grau de doentes fibromiálgicos apresentam um risco oito vezes superior de desenvolver esta síndrome em relação à população em geral. Estão ainda descritos polimorfismos específicos no gene codificador do transportador da serotonina e na enzima catecol-O-metiltransferase, que inactiva catecolaminas, e que têm sido associados a esta doença. Adicionalmente, foram ainda demonstradas alterações na actividade basal e estimulatória de vários eixos neuroendócrinos e desregulação do sistema nervoso autónomo. Os factores psicológicos têm uma grande influência na expressão clínica da FM e problemas associados. ${ }^{4,5}$

Apesar do reconhecimento e compreensão crescentes da FM, a sua heterogeneidade, complexidade e a interacção de factores neurobiológicos com aspectos psicossociais e comportamentais, tornam a abordagem e tratamento desta síndrome um desafio.

Assim, pretende-se rever, com base na evidência, e sistematizar a informação recentemente publicada sobre a abordagem terapêutica do doente com FM.

\section{MÉTODOS}

Foi realizada uma pesquisa na Medline e em sites de Medicina Baseada na Evidência (National Guideline Clearinghouse, Cochrane, Dare, Bandolier) de normas de orientação clínica (NOC), meta-análises (MA), revisões sistemáticas (RS) e estudos originais, em língua inglesa e portuguesa, publicados entre 1998 e Setembro de 2008. Utilizaram-se as palavras-chave: " $F i$ bromyalgia" e "Therapeutics".

Os critérios utilizados para inclusão de artigos nesta revisão foram os seguintes:

1) População: pessoas com diagnóstico de FM, de acordo com os critérios do Colégio Americano de Reumatologia (1990);

2) Intervenção e comparação: intervenções farmacológicas ou não-farmacológicas versus placebo ou outras intervenções farmacológicas ou não-farmacológicas;

3) Resultados:

- alterações na gravidade da dor, avaliada através da escala analógica da dor ou pelo número de pontos dolorosos (avaliados manualmente) ou pela avaliação do limiar de dor (utilizando um dolorímetro);

- alterações na qualidade do sono ou fadiga, avaliados por instrumentos auto-administrados;

- funcionalidade, avaliada por métodos validados como o Questionário de Impacto da Fibromialgia (QIF);

- perturbações do humor, avaliadas quer por sub-escalas do FIQ, quer por métodos validados, como o In- 
ventário de Depressão de Beck ou a Escala de Hamilton.

Obtiveram-se igualmente artigos pertinentes, e que cumpriam os critérios de inclusão, relacionados com os previamente consultados. Foram também pesquisadas recomendações de sociedades científicas, tendo sido seleccionadas aquelas em que é analisado o nível de evidência.

Foi utilizada a taxonomia SORT para avaliação do nível de evidência e força de recomendação. Segundo esta taxonomia, a qualidade do estudo está subdividida em três Níveis de Evidência (NE): NE 1 - estudos de boa qualidade, evidência orientada para o doente; $\mathrm{NE}$ 2 - estudos de qualidade limitada, evidência orientada para o doente; e NE 3 - outra evidência. A Força de Recomendação está divida em três graus: Força de recomendação A - provas consistentes de boa qualidade, orientadas para o doente; Força de recomendação B provas inconsistentes ou de qualidade limitada, orientadas para o doente; e Força de recomendação C - outros tipos de provas (provas orientadas para a doença, consensos, opiniões de peritos, extrapolações). ${ }^{6}$

\section{RESULTADOS}

Da pesquisa efectuada foram identificados 156 artigos. Destes, foram excluídos os artigos repetidos, os artigos que não cumpriam os critérios de inclusão e os estudos originais que foram alvo de análise em NOC, MA e RS incluídas nesta revisão. Foram incluídos no estudo duas NOC, três MA, onze RS e quatro estudos originais (Figura 1).

A abordagem da FM requer uma avaliação compreensiva da dor, funcionalidade e contexto psicossocial do doente. ${ }^{7}$ Os objectivos do tratamento incluem o alívio da dor, a melhoria da qualidade do sono, da fadiga, do bem-estar, da capacidade funcional e das perturbações ansiosas / depressivas associadas. ${ }^{4}$

Desta forma, sendo a FM polissintomática e heterogénea, e na ausência de um tratamento que actue em todos os sintomas, um tratamento optimizado irá requerer uma abordagem multidisciplinar individualizada, com uma associação de modalidades terapêuticas farmacológicas e não farmacológicas. Várias revisões e normas de orientação clínica recomendam a utilização de um tratamento multidisciplinar mas salientam a ausência de estudos de elevada qualidade nesta área. ${ }^{4,7,8}$

\section{Transmissão do diagnóstico e educação do doente} Uma etapa decisiva na abordagem do doente com FM consiste na transmissão do diagnóstico. É fundamental proporcionar informação assimilável ao doente e à sua família, assegurando-lhes que os sintomas são reais e não produto da sua imaginação, promovendo uma atitude positiva face aos mesmos e às propostas terapêuticas.

É aconselhável uma abordagem compreensiva, centrada no doente, com base numa relação médico-doente efectiva, de forma a avaliar o impacto da doença nas actividades sociais, ocupacionais ou em outras áreas importantes, e permitir conhecer e reconverter as concepções incorrectas sobre as causas dos sintomas e os receios sobre a afecção da capacidade funcional. O doente deverá ser educado sobre o carácter benigno do problema e a improbabilidade de deformação, deterioração física ou morte. Deverá ser reforçado que uma melhoria significativa dos sintomas raramente ocorre sem a sua participação activa, numa verdadeira

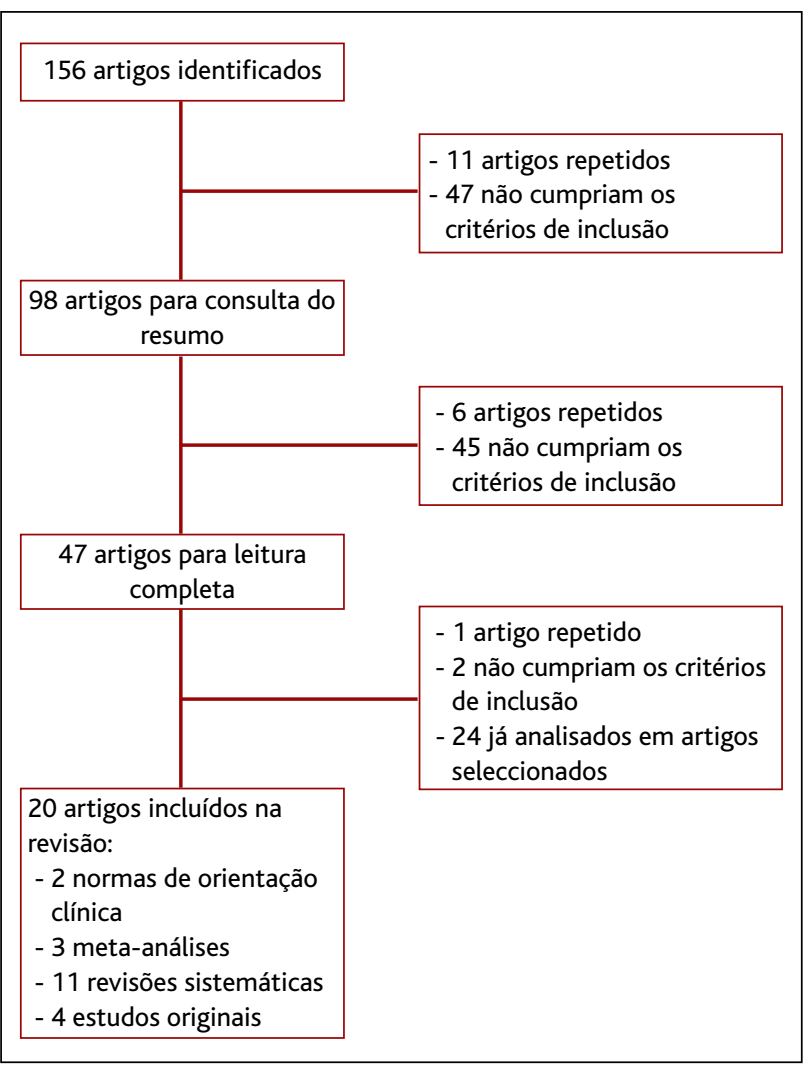

Figura 1. Processo de obtenção dos artigos incluídos na revisão 
aliança médico-doente. ${ }^{9}$

Apesar da dúvida de que a transmissão de um diagnóstico de FM pudesse afectar adversamente os doentes, um estudo deWhite et al. demonstrou uma melhoria na sintomatologia e na satisfação dos doentes e uma redução do consumo de consultas no Médico de Família e em cuidados hospitalares após a transmissão do diagnóstico (NE 2). ${ }^{10}$

De acordo com as NOC da American Pain Society (APS) de 2005 e uma revisão sistemática, há forte evidência de que programas de educação intensiva dos doentes são um tratamento eficaz na FM. Esses programas educacionais, que foram avaliados nos estudos randomizados e controlados incluídos, foram habitualmente actividades em grupo, incluindo palestras, demonstrações, discussão em grupo e fornecimento de material escrito ao longo de várias sessões (variando de 6 a 17). Mostrou-se benefício em um ou mais dos outcomes avaliados incluindo qualidade do sono, fadiga $\mathrm{e}$ sensação de bem-estar e essas alterações foram mantidas durante 3 a 12 meses. Um programa multidisciplinar de educação conduzido ao longo de um dia e meio demonstrou melhorias, após um mês, na gravidade da dor, fadiga, rigidez, ansiedade, depressão e sensação de bem-estar (NE 1).,11

\section{Tratamento farmacológico}

$\mathrm{O}$ tratamento farmacológico da FM tem sido baseado essencialmente em fármacos com actuação no sistema nervoso central (SNC). Apesar de muitos deles serem utilizados como anti-depressivos, relaxantes musculares ou anti-convulsivantes, estes fármacos afectam vários neurotransmissores (por exemplo: serotonina, noradrenalina, substância P) que desempenham um espectro alargado de funções no SNC incluindo modulação da sensação de dor e tolerância. ${ }^{4}$

Contudo, apesar da diversidade de opções farmacológicas estudadas, apenas a pregabalina é actualmente aprovada pela United States Food and Drug Administration para o tratamento da FM. ${ }^{9}$

\section{Anti-depressivos tricíclicos}

Os antidepressivos tricíclicos (ADTR) foram os primeiros fármacos a serem intensamente estudados para o tratamento da FM. ${ }^{9}$ O seu mecanismo de acção analgésica é incerto mas relaciona-se provavelmente com a inibição dual da recaptação neurohormonal, com maior impacto na noradrenalina. Existe ainda alguma evidência que os ADTR potenciam o sistema opióide endógeno e possuem outras propriedades farmacológicas, tais como a inibição da recaptação da dopamina, antagonismo NMDA e bloqueio de canais iónicos. ${ }^{5}$

A amitriptilina é o ADTR mais extensamente estudado. É fortemente recomendada para o tratamento da FM pela European League Against Rheumatism (EULAR), 2008 e pela APS. .11

Duas meta-análises publicadas evidenciaram melhoria moderada nos doentes fibromiálgicos tratados com amitriptilina (doses tituladas de 25 a $50 \mathrm{mg} / \mathrm{dia}$ ), em comparação com o placebo, para os outcomes gravidade da dor, bem-estar geral e sono. Mais especificamente, Arnold et al, relataram uma resposta clínica significativa à amitriptilina em $25-37 \%$ dos doentes, enquanto que na segunda meta-análise (O'Malley et al) relatou-se uma probabilidade quatro vezes superior de os doentes referirem melhoria global (em comparação com o placebo) e redução moderada dos sintomas individuais, semelhante à encontrada por Arnold et al. A melhoria observada foi mais acentuada na avaliação da qualidade do sono, enquanto que os efeitos mais modestos foram observados na rigidez e na gravidade da dor. Desta forma, o efeito benéfico obtido poderá ser atribuído, parcialmente, às propriedades sedativas destes agentes. ${ }^{12,13}$

Contudo, a maioria dos estudos incluídos foi de curta duração - 6 a 12 semanas. O único estudo prospectivo realizado com duração de 6 meses, no qual 208 doentes foram tratados com amitriptilina ou ciclobenzaprina ou placebo, mostrou que a melhoria sintomática inicial observada às 6-12 semanas não se verificou às 26 semanas. Associadamente, os ensaios apresentam tamanho amostral reduzido e não foram utilizadas as doses terapêuticas standard para a depressão. Para além disso, apesar da evidência de prevalências elevadas de depressão nos doentes com FM e o possível significado prognóstico de depressão prévia ou actual, nenhum estudo controlado avaliou sistematicamente os doentes para um diagnóstico de perturbação do humor (NE 2). . $^{12,13}$

Quando utilizada, a dose inicial de amitriptilina deverá ser de 5-10 mg 1 a 3 horas antes de deitar, de forma a minimizar efeitos laterais que ocorrem mais fre- 
quentemente com doses mais elevadas. A dose poderá ser aumentada $5 \mathrm{mg}$ a cada duas semanas. A dose final deverá ser ajustada ponderando eficácia versus efeitos laterais, mantendo a dose mais baixa possível. ${ }^{5}$ Os efeitos anti-colinérgicos, anti-histaminérgicos e anti- $\alpha$ -adrenégicos condicionam uma variedade de efeitos laterais indesejáveis incluindo: xerostomia, sedação, aumento de peso, retenção urinária, obstipação e taquicardia, que podem comprometer a tolerabilidade e a aceitabilidade deste fármaco. Doentes com problemas cardíacos ou glaucoma de ângulo fechado não deverão tomar ADTR; doentes idosos não deverão tomar amitriptilina devido ao seu risco elevado de efeitos laterais anti-colinérgicos. ${ }^{5}$ A nortriptilina, apesar de não ter sido avaliada em ensaio aleatorizado e controlado (EAC), tem um perfil de efeitos laterais mais favorável que a amitriptilina e pode também ser útil requerendo contudo dosagens ligeiramente superiores (NE 3 ). ${ }^{9}$

A ciclobenzaprina partilha a estrutura tricíclica da amitriptilina e da nortriptilina, não tendo contudo acção anti-depressiva, mas actuando a nível central como relaxante muscular esquelético. De acordo com a NOC da APS, há forte evidência para a utilização deste fármaco no tratamento da FM. ${ }^{11}$ Uma meta-análise publicada sobre o tratamento com ciclobenzaprina (10 a 40 $\mathrm{mg} / \mathrm{dia})$, e que só encontrou cinco estudos de qualidade adequada para incluir na análise, concluiu que os doentes tratados com este fármaco têm uma probabilidade três vezes superior de referir melhoria do estado geral e melhorias moderadas nos sintomas individuais, particularmente no sono. Não foram observados resultados estatisticamente significativos na fadiga e nos pontos dolorosos (NE 2). ${ }^{14}$

\section{Inibidores selectivos da recaptação da serotonina}

Os inibidores selectivos da recaptação da serotonina (ISRS), pela sua capacidade de aumentar a disponibilidade da serotonina na sinapse neuronal, têm sido estudados no tratamento da FM. ${ }^{9}$

Foram conduzidos quatro EAC para avaliar a eficácia da fluoxetina com resultados discordantes. Um primeiro estudo, envolvendo 42 doentes, não mostrou benefícios na sua utilização na dose de $20 \mathrm{mg} /$ dia em comparação com o placebo durante o período de follow-up de 6 semanas. Num segundo estudo, com 19 participantes, a mesma dose melhorou a dor e outros outcomes avaliados. Nesse estudo, a associação de 20 mg de fluoxetina de manhã com 25 mg de amitriptilina ao deitar foi mais eficaz que cada um dos fármacos isoladamente e ambos foram mais eficazes que o placebo. Resultados similares foram encontrados com a fluoxetina $(20 \mathrm{mg}$ ) em associação com a ciclobenzaprina $(10 \mathrm{mg})$. Um estudo mais recente $(\mathrm{n}=60)$, randomizado, controlado, com dupla ocultação e com dose flexível de fluoxetina ( $20 \mathrm{mg}$ até um máximo de $80 \mathrm{mg}$ ) evidenciou melhoria nos doentes tratados com este fármaco no score global do QIF e nos subscores para dor, fadiga e depressão. A melhoria na dor e funcionalidade foi independente das alterações do humor.

Considerando estes quatro estudos, a APS conclui haver evidência moderada para a utilização de fluoxetina, em monoterapia ou em associação a tricíclicos, para o alívio da dor na fibromialgia. ${ }^{11}$ Por seu lado, a EULAR incluiu na sua revisão apenas o estudo onde é feita comparação com a amitriptilina e o estudo com doses flexíveis de fluoxetina, por se tratar de estudos com qualidade moderada a elevada, concluindo haver forte evidência para a sua recomendação. ${ }^{7}$

Uma meta-análise (Arnold et al), de 2000, apenas incluiu os dois primeiros estudos citados, realçando que a maior eficácia observada com a terapêutica combinada poder-se-á dever a uma interacção farmacocinética entre a fluoxetina e os ADTR, interacção esta que tipicamente aumenta substancialmente os níveis plasmáticos dos últimos (NE 2). ${ }^{12}$ Três revisões sistemáticas publicadas concluem haver evidência moderada para a utilização da fluoxetina, com base nos quatros estudos originais já mencionados (NE 2). ${ }^{5,9,15}$

Relativamente a outros ISRS, os estudos realizados (citalopram, paroxetina, fluvoxamina) têm sido limitados, com baixa qualidade metodológica ou com resultados inconclusivos. $4,5,9,12$

A maior especificidade farmacológica dos ISRS relativamente aos ADTR, torna-os mais bem tolerados. Os efeitos laterais mais frequentes incluem náuseas, sedação, cefaleias, diminuição da líbido e disfunção sexual. ${ }^{5}$

\section{Inibidores da recaptação da serotonina e da noradrenalina}

Uma vez que a associação de fluoxetina com amitriptilina se mostrou eficaz na FM, colocou-se a hipótese 
de que fármacos que inibissem duplamente a serotonina e a noradrenalina fossem mais eficazes que um fármaco que actue predominantemente só num dos neurotransmissores. ${ }^{5}$ Assim, a duloxetina, o milnaciprano e a venlafaxina foram estudados em doentes fibromiálgicos.

Relativamente à duloxetina, um inibidor dual, a APS considera haver evidência moderada para a sua utilização, fundamentando-se num EAC, multicêntrico e de elevada qualidade metodológica. Nesse estudo, que envolveu 207 doentes, com duração de 12 semanas, foi utilizada uma dose de $60 \mathrm{mg} /$ dia de duloxetina, tendo-se verificado uma diminuição no número de pontos dolorosos, um aumento no limiar de pressão que desperta a dor e uma melhoria no QIF relativamente ao placebo. $^{11}$

A EULAR incluiu também um outro EAC, multicêntrico, com duração de 12 semanas, publicado posteriormente, no qual 354 participantes foram randomizados para duloxetina $60 \mathrm{mg}$ numa administração diária, duloxetina $60 \mathrm{mg} /$ dia em administração bipartida e placebo. Não se verificaram diferenças estatisticamente significativas entre a administração unidiária e a bipartida e ambos os grupos apresentaram melhoria significativa na redução da gravidade da dor comparativamente ao placebo. Em ambos os estudos a melhoria na dor foi independente das alterações do humor. Efeitos laterais encontrados incluíram insónia, xerostomia, obstipação, aumento da frequência cardíaca, elevação da aspartato-aminotransferase, da creatina cinase e do colesterol. Considera, assim, haver evidência de elevada qualidade que suporte a recomendação da utilização de duloxetina em doente com FM. ${ }^{7}$

Relativamente às revisões sistemáticas encontradas, foram incluídos os dois estudos já referidos, chegando a conclusões similares. ${ }^{5,9,15}$

Em 2008 foi publicado um terceiro estudo original que pretendeu avaliar a eficácia e a segurança da duloxetina no tratamento da FM em doentes com e sem perturbação depressiva major. Tratou-se de um EAC, multicêntrico, no qual 520 participantes foram randomizados para duloxetina $(20 \mathrm{mg} / \mathrm{dia}, 60 \mathrm{mg} / \mathrm{dia}$ ou 120 $\mathrm{mg} / \mathrm{dia}$ ) ou placebo, administrados uma vez ao dia durante seis meses (após o terceiro mês, o grupo que estava a fazer $20 \mathrm{mg} /$ dia de duloxetina foi titulado para $60 \mathrm{mg}$ /dia). A utilização de duloxetina em doses de 60 $\mathrm{mg} /$ dia e $120 \mathrm{mg} /$ dia foi significativamente mais eficaz que o placebo na redução da gravidade da dor após 3 e 6 meses de tratamento. Comparativamente ao placebo, a duloxetina em ambas as dosagens reduziu a gravidade da dor após a primeira semana de tratamento. À semelhança do que foi verificado nos estudos anteriores, a melhoria na gravidade da dor foi independente da presença de perturbação depressiva major, demonstrando-se que grande parte do efeito da duloxetina na dor é directo e não mediado através de melhorias nos sintomas depressivos. Os efeitos laterais foram semelhantes aos já descritos (NE 1). ${ }^{16}$

O milnaciprano, um inibidor dual da recaptação, com maior actividade inibitória noradrenégica, é recomendado com evidência moderada pela APS e com forte evidência pela EULAR. Apenas foi conduzido um estudo que avaliou o milnaciprano. Tratou-se de um EAC, multicêntrico, com duração de 12 semanas, no qual 125 participantes receberam milnaciprano $100 \mathrm{mg}, 200 \mathrm{mg}$ ou placebo. Demonstrou-se uma melhoria global no QIF e na dor avaliada através da escala analógica relativamente ao placebo. Os efeitos laterais foram semelhantes aos descritos com a duloxetina. Uma dosagem de $100 \mathrm{mg}$ duas vezes por dia foi mais eficaz que $200 \mathrm{mg}$ administrados uma vez por dia (NE 1)..$^{7,11}$

Contrariamente aos ADTR, a duloxetina e o milnaciprano foram utilizados em doses similares ou superiores às recomendadas para o tratamento anti-depressivo, conferindo-lhes a vantagem adicional de tratarem depressões co-mórbidas que comummente se verificam nos doentes com FM. ${ }^{5,9}$

Em duas revisões sistemáticas sobre tratamento da FM foram incluídos três estudos sobre a utilização da venlafaxina: na dosagem de $75 \mathrm{mg} /$ dia não apresentou benefícios em relação ao placebo num estudo randomizado e controlado, tendo sido útil em dois estudos de pequenas dimensões utilizando doses mais elevadas (NE 2). ${ }^{4,15}$

\section{Anti-convulsivantes}

A pregabalina e o gabapentina são fármacos com mecanismos de acção similares. Actuam na subunidade $\alpha_{2} \delta$ de canais de cálcio dependentes da voltagem, reduzindo o influxo de cálcio durante a despolarização e diminuindo a libertação de glutamato, noradrenalina e substância P. ${ }^{5}$ 
A pregabalina é, actualmente, o único fármaco aprovado pela United States Food and Drug Administration para o tratamento da FM. ${ }^{9}$

A EULAR e a APS consideram haver forte e moderada evidência, respectivamente, para a sua utilização na FM. Estas recomendações fundamentam-se num EAC, multicêntrico, com duração de 8 semanas, envolvendo 529 doentes, no qual foram comparadas várias dosagens do fármaco (150, 300, $450 \mathrm{mg} /$ dia). A pregabalina (450 mg/dia) reduziu de forma significativa a gravidade da dor comparativamente ao placebo e um maior número de doentes relataram melhoria em mais de $50 \%$ da dor. Melhorou ainda o sono, a fadiga e a qualidade de vida $(300 \mathrm{mg} /$ dia, $450 \mathrm{mg} / \mathrm{dia})$. O fármaco foi bem tolerado; tonturas e sonolência ocorreram mais frequentemente no grupo tratado com pregabalina mas originaram baixas taxas de descontinuação. ${ }^{7,11}$

As revisões sistemáticas encontradas também fundamentam a recomendação deste fármaco com base neste estudo. ${ }^{4,5,9}$

Posteriormente, foi publicado um EAC, multicêntrico, que pretendeu avaliar a eficácia da pregabalina, em monoterapia, quanto à durabilidade do efeito na gravidade da dor na FM. O estudo, com duração de seis meses, demonstrou a durabilidade do efeito na redução da dor, assim como melhoria do sono, fadiga, funcionalidade e bem-estar, com baixas taxas de descontinuação por efeitos laterais (NE 1). ${ }^{17}$

Duas revisões sistemáticas avaliaram a utilização da gabapentina na FM. Incluíram um EAC $(\mathrm{n}=150)$ no qual foram utilizadas dosagens de $1200 \mathrm{mg} /$ dia a 2400 $\mathrm{mg} /$ dia de gabapentina, tendo-se verificado ser mais eficaz que o placebo na melhoria da dor $(51 \%$ vs $31 \%$, melhoria clínica definida como melhoria em mais de $30 \%$ da dor), qualidade do sono e QIF (NE 1)..$^{5,9}$

\section{Analgésicos}

O tramadol é um analgésico opióide sintético, de actuação central, que se liga aos receptores opióides $\mu$ e que inibe modestamente a recaptação da noradrenalina e da serotonina. ${ }^{9}$

De acordo com uma revisão da literatura incluída na NOC da EULAR, há forte evidência para a utilização do tramadol nos doentes com FM. A APS considera haver evidência moderada. A base destas recomendações foram os benefícios demonstrados em dois estudos rando- mizados e controlados de qualidade moderada a elevada. Um primeiro estudo, incluindo 100 participantes, relatou uma diminuição nos scores da escala analógica da dor e no alívio da dor com o tratamento com tramadol (50 mg titulados ao longo de 14 dias, atingindo um máximo de $400 \mathrm{mg} /$ dia, durante 6 semanas). Um estudo recente comparou uma combinação de $37,5 \mathrm{mg}$ tramadol / $325 \mathrm{mg}$ de paracetamol (até $75 \mathrm{mg}$ tramadol / $650 \mathrm{mg}$ paracetamol, quatro vezes por dia) com o placebo em 315 doentes. As taxas de descontinuação e os scores da dor e do QIF foram estatisticamente melhores no grupo tratado com a associação de fármacos. ${ }^{7,11}$ A EULAR ressalva, contudo, que o tramadol deve ser usado com alguma precaução tendo em conta a possibilidade de síndrome de privação com a sua descontinuação e o risco de abuso e dependência. ${ }^{6}$ Conclusões semelhantes foram obtidas nas revisões sistemáticas encontradas. ${ }^{4,5,9}$

Apesar de o uso de anti-inflamatórios não esteróides (AINEs) para a FM ser frequente, a evidência é insuficiente para a sua recomendação. ${ }^{7,11}$ Estudos randomizados e controlados com ibuprofeno e naproxeno não evidenciaram melhorias dos sintomas em relação ao placebo. ${ }^{4,5,9}$

A recomendação da não utilização de opióides de elevada potência é baseada em opiniões de peritos, considerando os seus efeitos laterais significativos e a ausência de estudos clínicos. ${ }^{5,7,11}$

\section{Outros fármacos}

A EULAR e uma revisão sistemática (Arnold et al) avaliaram a utilização de dois inibidores da monoaminoxidase-A - moclobemida e pirlindole - na FM. ${ }^{7,12}$ Analisaram dois EAC. Num EAC, com duração de 12 semanas, 130 participantes com co-morbilidades psiquiátricas foram randomizados para moclobemida $(450-600$ $\mathrm{mg} /$ dia), amitriptilina ou placebo. No grupo tratado com moclobemida não houve melhoria na gravidade da dor em relação ao placebo. Num estudo com duração de quatro semanas, e 100 participantes, o pirlindole (75 $\mathrm{mg} / \mathrm{dia}$ ) foi bem tolerado e produziu melhorias estatisticamente significativas na gravidade da dor relativamente ao placebo, o que leva a EULAR a o recomendar para o tratamento da FM. Arnold et al salientam a necessidade de estudos complementares.

O tropisetron, um antagonista dos receptores 5-HT3 da serotonina, foi avaliado em estudos clínicos com li- 
mitações metodológicas e resultados inconsistentes mas mostrou um efeito positivo na dor com uma dose de 5 mg num dos estudos. ${ }^{5,7}$

O pramipexole, um agonista dopaminérgico, mostrou-se moderadamente eficaz em alguns doentes com FM. Um estudo concluiu que este fármaco reduziu a dor a e fadiga e melhorou a funcionalidade em relação ao placebo. No entanto, considerando que os doentes que participaram no estudo permaneceram com as suas medicações habituais, incluindo opióides, o uso do pramipexole poderá ser ponderado em doentes refractários a múltiplas terapêuticas mas são necessários mais estudos para avaliar a sua eficácia e segurança., ${ }^{5,7}$

O único estudo controlado que avaliou o efeito dos corticoesteróides na FM revelou que $10 \mathrm{mg}$ de prednisolona diárias não são benéficos. ${ }^{4}$

De igual modo, não há evidência de que as benzodiazepinas ou sedativos não-benzodiazepínicos sejam eficazes em doentes com FM para além do seu papel nas perturbações do sono. ${ }^{4}$

Não há evidência de estudos randomizados e controlados para a utilização de hormonas tiróideias, dehidroepiandrosterona, melatonina ou calcitonina. Modificações dietéticas, suplementos nutricionais, magnésio e outros suplementos vitamínicos não foram adequadamente avaliados na FM. ${ }^{4}$

\section{Tratamento não farmacológico}

\section{Exercício físico}

A prática de exercício físico é frequentemente recomendada a doentes com FM com base no pressuposto de que estes doentes apresentam um descondicionamento muscular e um maior risco de microtraumatismo muscular que acarreta um aumento da dor. ${ }^{9}$

A revisão sistemática incluída nas NOC da EULAR, e que incluiu estudos publicados até 2005, concluiu que a recomendação para a prática de exercício físico é baseada em opiniões de peritos em associação com alguma evidência experimental, revisões e NOC prévias, incluindo as da APS. Refere que relativamente ao exercício aeróbico, a maioria dos estudos não são aleatorizados, com excepção de dois estudos randomizados e controlados. Foi encontrado um possível efeito positivo na gravidade da dor e limitado efeito na função. Dos estudos que avaliaram o efeito do exercício de força muscular apenas um foi realizado com dupla-oculta- ção, não se verificando diferenças na gravidade da dor e função (NE 1).?

Uma revisão sistemática (van Koulil et al), de 2006, salienta igualmente as limitações metodológicas dos estudos realizados, concluindo que os efeitos benéficos do exercício físico parecem limitados, especialmente a longo prazo (NE 1). ${ }^{18}$

Uma revisão sistemática da Cochrane publicada em 2008, com 34 ensaios clínicos incluídos, avaliou os efeitos do exercício físico, incluindo exercício cardio-respiratório (aeróbico), de força muscular e/ou de flexibilidade, no bem-estar geral, função física, gravidade da dor e pontos dolorosos em doentes com FM. Concluiu haver evidência moderada de que a prática de exercício aeróbico, em níveis de intensidade recomendados, tem efeitos positivos no bem-estar geral, função física e possivelmente na gravidade da dor e pontos dolorosos. Os exercícios de força muscular e flexibilidade permanecem sub-avaliados (NE 1)..$^{19}$

Um estudo recente (incluindo 207 doentes), randomizado, que pretendeu avaliar os benefícios da prática de exercício físico aeróbico e de flexibilidade na FM concluiu que a prática de caminhadas progressivas e exercícios de flexibilidade melhoram o estado funcional, a gravidade da dor, a fadiga, a depressão e a autoeficácia (NE 2). ${ }^{20}$

\section{Balneoterapia}

O efeito da balneoterapia foi avaliado na revisão sistemática incluída nas NOC da EULAR. Incluiu cinco estudos aleatorizados publicados. Três dos estudos incluíram exercício físico na intervenção (dois com efeitos positivos na função e dois com efeitos positivos na gravidade da dor). Os estudos que não incluíram exercício físico mostraram um efeito positivo na gravidade da dor e função. Assim, foi concluído que a balneoterapia, com ou sem exercício, é moderadamente eficaz na FM. ${ }^{7}$ Uma recomendação similar é feita pela APS. ${ }^{11}$

\section{Terapia cognitivo-comportamental}

Relativamente à utilização de terapia cognitivo-comportamental para o tratamento da fibromialgia, duas RS relatam melhoria na gravidade da dor, fadiga, humor e função nos doentes que receberam esta intervenção (NE 2). ${ }^{21,22}$ Com base nestas RS, a APS refere haver forte evidência para a sua utilização nos doentes com FM. ${ }^{11}$ 


\begin{tabular}{|c|c|c|c|}
\hline Intervenção & Efeitos na FM & Comentários & $\begin{array}{c}\text { Força de } \\
\text { Recomendação }\end{array}$ \\
\hline Amitriptilina & $\begin{array}{l}\text { - Efeito benéfico especialmente se } \\
\text { perturbações do sono associadas }\end{array}$ & $\begin{array}{l}\text { - Sem evidência de melhoria sintomática } \\
\text { a médio prazo } \\
\text { - Estudos realizados com limitações } \\
\text { metodológicas } \\
\text { - Os efeitos benéficos poderão ser atribuídos, } \\
\text { em parte, às suas propriedades sedativas }\end{array}$ & B \\
\hline Ciclobenzaprina & $\begin{array}{l}\text { - Efeitos benéficos no sono e na gravidade } \\
\text { da dor }\end{array}$ & $\begin{array}{l}\text { - Ausência de estudos que avaliem eficácia a } \\
\text { médio/longo prazo } \\
\text { - Estudos realizados com limitações } \\
\text { metodológicas }\end{array}$ & B \\
\hline Fluoxetina & $\begin{array}{l}\text { - Efeitos benéficos na gravidade da dor, fadiga } \\
\text { e depressão } \\
\text { - Efeito benéfico independente das alterações } \\
\text { do humor } \\
\text { - Associação de fluoxetina com ADTR: maior } \\
\text { eficácia que cada fármaco isolado }\end{array}$ & $\begin{array}{l}\text { - Ausência de estudos que avaliem eficácia a } \\
\text { médio/longo prazo } \\
\text { - Um estudo com resultados discordantes } \\
\text { - Tratamento de perturbação depressiva } \\
\text { associada } \\
\text { - Maior eficácia da associação com ADTR: } \\
\text { aumento dos níveis plasmáticos dos ADTR? }\end{array}$ & B \\
\hline Duloxetina & $\begin{array}{l}\text { - Melhoria da gravidade da dor } \\
\text { - Efeito benéfico independente das alterações } \\
\text { do humor }\end{array}$ & $\begin{array}{l}\text { - Efeito mantido aos } 6 \text { meses de tratamento } \\
\text { - Tratamento de perturbação depressiva } \\
\text { associada }\end{array}$ & A \\
\hline Milnaciprano & - Melhoria da gravidade da dor & $\begin{array}{l}\text { - Apenas avaliado em } 1 \text { EAC } \\
\text { - Tratamento de perturbação depressiva } \\
\text { associada }\end{array}$ & B \\
\hline Pregabalina & $\begin{array}{l}\text { - Melhoria da gravidade da dor, sono, fadiga e } \\
\text { funcionalidade }\end{array}$ & - Efeito mantido aos 6 meses de tratamento & A \\
\hline Gabapentina & $\begin{array}{l}\text { - Melhoria da gravidade da dor e qualidade } \\
\text { do sono }\end{array}$ & - Apenas avaliado em $1 \mathrm{EAC}$ & B \\
\hline Tramadol & $\begin{array}{l}\text { - Melhoria da dor } \\
\text { - Efeito benéfico também verificado com a } \\
\text { associação com paracetamol }\end{array}$ & $\begin{array}{l}\text { - Usar com precaução: síndrome de privação, } \\
\text { risco de abuso e dependência }\end{array}$ & A \\
\hline Pirlindole & - Melhoria da dor & - Apenas avaliado em 1 EAC de curta duração & $\mathrm{B}$ \\
\hline \multicolumn{3}{|c|}{$\begin{array}{l}\text { Sem evidência demonstrada: citalopram, paroxetina, fluvoxamina, venlafaxina, AINEs, opióides de elevada } \\
\text { potência, corticoesteróides, benzodiazepinas, sedativos não-benzodiazepínicos, hormonas tiróideias, } \\
\text { dehidroepiandrosterona, melatonina, calcitonina, modificações dietéticas, suplementos nutricionais, } \\
\text { suplementos vitamínicos. }\end{array}$} & C \\
\hline
\end{tabular}

No entanto, na RS da EULAR, salienta-se as limitações metodológicas dos estudos realizados, com ausência de aleatorização e manutenção da terapêutica habitual nos doentes avaliados, concluindo que a recomendação para a sua utilização é baseada em opinião de peritos. ${ }^{6}$ Conclusão similiar foi obtida por van Koulil et al (NE 1). ${ }^{18}$

\section{Medicinas alternativas e complementares}

Uma revisão de 2003 que avaliou a utilização de medicinas alternativas e complementares na FM concluiu que, das várias modalidades consideradas e que incluíram acupunctura, homeopatia, terapias de base biológica (ex.: suplementos nutricionais), modificações dietéticas, terapias energéticas (ex.: terapia magnéti- 


\begin{tabular}{|c|c|c|c|}
\hline Intervenção & Efeitos na FM & Comentários & $\begin{array}{c}\text { Força de } \\
\text { Recomendação }\end{array}$ \\
\hline Exercício físico & $\begin{array}{l}\text { - Exercício físico aeróbico: melhoria da } \\
\text { gravidade da dor }\end{array}$ & $\begin{array}{l}\text { - Estudos realizados com limitações } \\
\text { metodológicas } \\
\text { - Resultados inconsistentes }\end{array}$ & B \\
\hline Balneoterapia & - Melhoria da dor e funcionalidade & $\begin{array}{l}\text { - Estudos realizados com limitações } \\
\text { metodológicas } \\
\text { - Resultados inconsistentes }\end{array}$ & B \\
\hline $\begin{array}{l}\text { Terapia } \\
\text { cognitivo- } \\
\text {-comportamental }\end{array}$ & $\begin{array}{l}\text { - Melhoria da gravidade da dor, fadiga, } \\
\text { humor e funcionalidade }\end{array}$ & $\begin{array}{l}\text { - Baseada em opinião de peritos } \\
\text { - Estudos realizados com limitações } \\
\text { metodológicas }\end{array}$ & C \\
\hline \multicolumn{3}{|c|}{$\begin{array}{l}\text { Sem evidência demonstrada: exercício físico de força muscular e flexibilidade, acupunctura, homeopatia, } \\
\text { terapias de base biológica (ex.: suplementos nutricionais), modificações dietéticas, terapias energéticas, } \\
\text { sistemas manipulativos de base corporal (ex.: quiroprática, massagens) e intervenções corpo-mente (ex.: } \\
\text { relaxamento, biofeedback e hipnoterapia) }\end{array}$} & C \\
\hline
\end{tabular}

ca), sistemas manipulativos de base corporal (ex.: quiroprática, massagens) e intervenções corpo-mente (ex.: relaxamento, biofeedback e hipnoterapia), nenhuma foi consistentemente eficaz. A acupunctura foi a modalidade com maior evidência de eficácia (NE 2). ${ }^{23}$

Uma revisão sistemática recente pretendeu avaliar a eficácia da acupunctura no tratamento sintomático da FM. Incluiu cinco estudos randomizados e controlados, ressalvando a sua qualidade metodológica frequentemente baixa. Três dos estudos mostraram resultados positivos mas de curta-duração, os outros dois estudos não mostraram benefícios. Todos os estudos positivos utilizaram electro-acupunctura. Assim sendo, concluiu-se que a noção de que a acupunctura é eficaz no tratamento da fibromialgia não é confirmada pelos resultados dos estudos clínicos, não havendo evidência para a sua recomendação (NE 1). ${ }^{24}$

\section{CONCLUSÕES}

A abordagem da FM, enquanto síndrome polissintomática e heterogénea, requer uma avaliação da dor, funcionalidade, contexto psicossocial e co-morbilidades associadas.

A sua natureza crónica implica a realização de tratamento a longo prazo e que inclui a educação e explicação da perturbação ao doente (Força de recomendação
A) e uma associação de medidas farmacológicas e não farmacológicas, discutidas com o doente, motivando a sua adesão, de acordo com a gravidade da dor, funcionalidade e sintomas associados tais como depressão, fadiga e perturbações do sono (Força de recomendação C).

Relativamente à terapêutica farmacológica (Quadro II), os anti-depressivos tricíclicos amitriptilina e ciclobenzaprina reduzem a gravidade da dor e, como apresentam igualmente um efeito benéfico nas perturbações do sono, poderão ser vantajosos nessas situações (Força de recomendação B).

A duloxetina (Força de recomendação A) e a fluoxetina (Força de recomendação B) têm efeitos benéficos na redução da dor e, ao contrário dos tricíclicos, são utilizados no tratamento da FM em doses similares ou superiores às recomendadas para o tratamento anti-depressivo, podendo ser vantajosas em doentes com patologia depressiva co-mórbida. O milnaciprano e o pirlindole foram avaliados em um estudo randomizado e controlado cada, com resultados promissores, sendo necessários mais estudos para avaliar de forma consistente a sua eficácia e segurança (Força de recomendação B).

A pregabalina (Força de recomendação A) e a gabapentina (Força de recomendação B) mostraram-se be- 
néficas na redução da dor e nas perturbações do sono em doentes com FM.

Quanto a fármacos analgésicos, há forte evidência para a utilização de tramadol na dor da FM (Força de recomendação A). Os AINEs não deverão ser utilizados como terapêutica de primeira linha (Força de recomendação B). Não é recomendada a utilização de corticoesteróides e de opióides de elevada potência (Força de recomendação C).

Em relação à terapêutica não farmacológica (quadro III), a prática de exercício físico aeróbico, de intensidade moderada, tem efeitos benéficos no bem-estar geral, na função física e na gravidade da dor (Força de recomendação B). A balneoterapia, com ou sem exercício, é eficaz na FM (Força de recomendação B). A evidência é insuficiente para a recomendação da prática de exercício de força muscular e de flexibilidade, terapia cognitivo-comportamental e medicinas alternativas e complementares, incluindo a acupunctura (Força de recomendação C).

Apesar de haver evidência da eficácia de certas terapêuticas na FM a curto prazo, apenas a duloxetina e a pregabalina demonstraram benefícios em estudos com duração de seis meses. Novos ensaios aleatorizados e controlados necessitam de serem conduzidos de forma a avaliar a eficácia, tolerabilidade e segurança a médio/longo prazo de vários tratamentos numa doença que é crónica.

Para além disso, vários sintomas e co-morbilidades frequentemente associados à FM têm sido inconsistentemente avaliados e poderão afectar a resposta ao tratamento e explicar a variabilidade nas respostas observadas em certos estudos. Concomitantemente, essa heterogeneidade condiciona a necessidade de programas terapêuticos multidisciplinares ou associando várias modalidades de tratamento, que têm sido sub-investigadas.

\section{REFERÊNCIAS BIBLIOGRÁFICAS}

1. Chakrabarty S, Zoorob R. Fibromyalgia. Am Fam Physician 2007 Jul 15; 76 (2): 247-54.

2. Branco JC. Fibromialgia. In: Cardoso A, Branco JC, Silva J, Cruz M, Costa MM, editores. Regras de Ouro em Reumatologia. Lisboa: DGS; 2005. p. 37-46.

3. Direcção-Geral da Saúde. Divisão de doenças genéticas, crónicas e geriátricas. Programa Nacional contra as doenças reumáticas. Lisboa: DGS; 2005. p. 43-7.
4. Goldenberg DL, Burckhardt C, Crofford L. Management of fibromyalgia syndrome. JAMA 2004 Nov 17; 292 (19): 2388-95.

5. Goldenberg D. Pharmacological treatment of fibromyalgia and other chronic musculoskeletal pain. Best Pract Res Clin Rheumatol 2007 Jun; 21(3):499-511.

6. Ebell MH, Siwek J, Weiss BD, Woolf SH, Susman J, Ewingman B, et al. Strength of Recommendation Taxonomy (SORT): a patient-centered approach to grading evidence in the medical literature. Am Fam Physician 2004 Feb 1; 69 (3):548-56.

7. Carville SF, Arendt-Nielsen S, Bliddal H, Blotman F, Branco JC, Buskila $D$, et al. EULAR evidence-based recommendations for the management of fibromyalgia syndrome. Ann Rheum Dis 2008 Apr; 67 (4): 536-41.

8. Karjalainen K, Malmivaara A, van Tulder M, Roine R, Jauhiainen M, Hurri $\mathrm{H}$, et al. Multidisciplinary rehabilitation for fibromyalgia and musculoskeletal pain in working age adults. Cochrane Database Syst Rev 2000; (2): CD001984.

9. Abeles $\mathrm{M}$, Solitar BM, Pillinger $\mathrm{MH}$, Abeles AM. Update on fibromyalgia therapy. Am J Med 2008 Jul; 121 (7): 555-61.

10. White KP, Nielson WR, Harth M, Ostbye T, Speechley M. Does the label "fibromyalgia" alter health status, function, and health service utilization? A prospective, within-group comparison in a community cohort of adults with chronic widespread pain. Arthritis Rheum 2002 Jun 15; 47 (3): 260-5.

11. Buckhardt CS, Goldenberg D, Crofford L, Gerwin R, Gowens S, Jackson $K$, et al. Guideline for the management of fibromyalgia syndrome pain in adults and children. Glenview, IL:American Pain Society; 2005. p. 109.

12. Arnold LM, Keck PE Jr, Welge JA. Antidepressant treatment of fibromyalgia: a meta-analysis and review. Psychosomatics 2000 Mar-Apr; 41 (2): 104-13.

13. O'Malley PG, Balden E, Tomkins G, Santoro J, Kroenke K, Jackson JL. Treatment of fibromyalgia with antidepressants: a meta-analysis. J Gen Intern Med 2000 Sep; 15 (9): 659-66.

14. Tofferi JK, Jackson JL, O'Malley PG. Treatment of fibromyalgia with cyclobenzaprine: a meta-analysis. Arthritis Rheum 2004 Feb 15; 51 (1): 9-13.

15. Arnold LM. Duloxetine and other antidepressants in the treatment of patients with fibromyalgia. Pain Med 2007 Sep; 8 Suppl 2: S63-74.

16. Russell IJ, Mease PJ, Smith TR, Kajdasz DK, Wohlreich MM, Detke MJ, et al. Efficacy and safety of duloxetine for treatment of fibromyalgia in patients with or without major depressive disorder: results from a 6month, randomized, double-blind, placebo-controlled, fixed-dose trial. Pain 2008 Jun; 136 (3): 432-44.

17. Crofford LJ, Mease PJ, Simpson SL, Young JP Jr, Martin SA, Haig GM, et al. Fibromyalgia relapse evaluation and efficacy for durability of meaningful relief (FREEDOM): a 6-month, double-blind, placebo-controlled trial with pregabalin. Pain 2008 Jun; 136 (3): 419-31.

18. van Koulil S, Effting M, Kraaimaat FW, van Lankveld W, van Helmond $\mathrm{T}$, Cats $\mathrm{H}$, et al. Cognitive-behavioural therapies and exercise programmes for patients with fibromyalgia: state of the art and future directions. Ann Rheum Dis 2007 May; 66 (5): 571-81.

19. Busch AJ, Barber KA, Overend TJ, Peloso PM, Schachter CL. Exercise for treating fibromyalgia syndrome. Cochrane Database Syst Rev 2007 Oct 17; (4): CD003786.

20. Rooks DS, Gautam S, Romeling M, Cross ML, Stratigakis D, Evans B, et 
al. Group exercise, education, and combination self-management in women with fibromyalgia. Arch Intern Med 2007 Nov; 167 (20): 2192200.

21. Williams DA. Psychological and behavioural therapies in fibromyalgia and related syndromes. Best Pract Res Clin Rheumatol 2003 Aug; 17 (4): 649-65.

22. Rossy LA, Buckelew SP, Dorr N, Hagglund KJ, Thayer JF, McIntosh MJ, et al. A meta-analysis if fibromyalgia treatment interventions. Ann Behav Med 1999 Spring; 21 (2): 180-91.

23. Holdcraft LC, Assefi N, Buchwald D. Complementary and alternative medicine in fibromyalgia and related syndromes. Best Pract Res Clin Rheumatol 2003 Aug; 17 (4): 667-83.

24. Mayhew E, Ernst E. Acupuncture for fibromyalgia: a systematic review of randomized clinical trials. Rheumatology 2007 May; 46 (5): 801-4.
Os autores declararam não possuir conflitos de interesses

\author{
ENDEREÇO PARA CORRESPONDÊNCIA \\ Paula Sofia Novais Gomes \\ Rua S. João, n. ${ }^{\circ} 12$ \\ Monte Largo - Azurém \\ 4800-064 Guimarães \\ E-mail: internos.infesta@gmail.com
}

Recebido em 22/09/2009

Aceite para publicação em 15/01/2010

\section{ABSTRACT}

\section{FIBROMYALGIA:THERAPEUTIC APPROACH}

Goal: To review and systematize the recently published data about the therapeutic management of the fibromyalgic patient. Data sources: National Guideline Clearinghouse, Cochrane, Dare, Bandolier e Medline.

Review methods: Search of guidelines, meta-analysis, systematic reviews and original studies written in english and portuguese, published between 1998 and September 2008, using the keywords: "Fibromyalgia" and "Therapeutics". Recommendations of scientific societies were also searched and those in which the level of evidence was analyzed were selected. 2 guidelines, 3 meta-analysis, 11 systematic reviews and 4 original studies were included. SORT taxonomy was used to attribute the level of evidence and strength of recommendation.

Results: The management of Fibromyalgia requires an assessment of pain severity, function, psychosocial context and associated morbidities. Regarding pharmacological therapy, there is strong evidence supporting the use of duloxetine, pregabalin and gabapentin for pain management. Moderate evidence supports the use of amitriptyline and cyclobenzaprine (specially if associated sleep disorders) and fluoxetine. Milnacipran and pirlindole showed promising results but further studies and needed. The positive effects reported with fluoxetine, duloxetine and milnacipran were independent of the presence of mood disorders and the dosages used were similar or higher than those recommended for antidepressive treatment, allowing the management of associated depression. As for non-pharmacological therapy, there is moderate evidence supporting aerobic exercise and heated pool treatment.

Conclusions: Multiple pharmacological and non-pharmacological treatments showed positive effects in the control of pain and related symptoms. In the absence of a treatment that targets all the symptoms of fibromyalgia, an optimized management will require an individualized multidisciplinary approach.

Keywords: Fibromyalgia; Therapeutics; Pain. 